\title{
CXC Chemokine Ligand 16 Promotes the Proliferation and Migration of Hodgkin and Reed-Sternberg Cells Via the PI3K/Akt/NF-кB Signaling Pathway
}

ISSN: 2637-773X

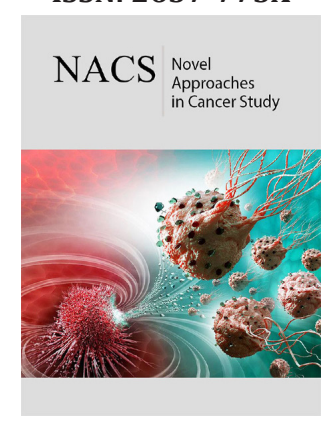

*Corresponding author: Fang Liu, Professor, School of Medicine, Department of Basic Medicine and Biomedical Engineering, Hebin Road, Foshan, Guangdong, 528000, China

Submission: 笽 August 11, 2021

Published: 僵 September 08, 2021

Volume 6 - Issue 2

How to cite this article: Yang Xun, Hongping Tang, Yu Pan, Dinghua Shen, Fang Liu, et al. CXC Chemokine Ligand 16 Promotes the Proliferation and Migration of Hodgkin and Reed-Sternberg Cells Via the PI3K/Akt/NF-кB Signaling Pathway. Nov Appro in Can Study. 6(2). NACS.000635. 2021

DOI: $10.31031 / \mathrm{NACS} .2021 .06 .000635$

Copyright@ Fang Liu, This article is distributed under the terms of the Creative Commons Attribution 4.0 International License, which permits unrestricted use and redistribution provided that the original author and source are credited.
Yang Xun ${ }^{1}$, Hongping Tang ${ }^{2}$, Yu Pan ${ }^{3}$, Dinghua Shen ${ }^{1}$, Hua Yang ${ }^{1}$, Yijun Liang ${ }^{1}$, Ruixue Wang ${ }^{1}$, Lixia Fan ${ }^{1}$, Hui Liu ${ }^{1}$, Yilong Ai ${ }^{1}$, Dahai Liu ${ }^{1}$ and Fang Liu ${ }^{1 *}$

${ }^{1}$ Department of Basic Medicine and Biomedical Engineering, China

${ }^{2}$ Department of Pathology, China

${ }^{3}$ Department of Stomatology, China

\begin{abstract}
Hodgkin's Lymphoma (HL) is a main type of lymphoma characterized by Hodgkin's ReedSternberg(H/RS) cells, which involves in many cytokines and chemokines. CXC Chemokine Ligand 16 (CXCL16) was reported to be expressed in various cancer types and was previously observed by our group to highly express in H/RS cells, yet the function and mechanism of CXCL16, as well as soluble CXCL16 (sCXCL16) on the phenotypes of HRS cells needs to be fully elucidated. To investigate the effects of CXCL16 on cell proliferation, migration, cell cycle, apoptosis, and immune-phenotypes of H/RS cells, an H/RS cell line (L428) transfected with CXCL16-overexpressed lentivirus vector was established and identified; besides, recombinant sCXCL16 was also applied on L428 cell lines in the present study. Results showed that both endogenously overexpressed CXCL16 and exogenously applied recombinant sCXCL16 protein were able to promote the proliferation and migration of L428 cells, and diminished cell apoptosis, while the immune phenotypes of L428 cells showed no significant changes. Blocking the interaction between the ligand CXCL16 and its unique receptor CXCR6 with antibody suppressed the above effects. As the pathways involved were concerned, results showed that CXCL16 upregulated the expression of several phosphorylated proteins of the PI3K/Akt and NF- $\mathrm{kB}$ signaling pathways, which were markedly inhibited by PI3K inhibitor LY294002 or CXCR6 antibody. The present study suggests that CXCL16 promotes the proliferation and migration of H/RS cell through its receptor CXCR6. The mechanism may involve activation of the PI3K/Akt/NF- $\mathrm{BB}$ signaling pathways.
\end{abstract}

Keywords: CXC chemokine ligand 16; Soluble CXCL16; Hodgkin 's lymphoma; Hodgkin reed-sternberg; CXCR6; Signaling pathway

\section{Background}

Hodgkin's Lymphoma (HL) is one of the most common malignant neoplasms affecting hematological and lymphoid systems. The leading causes of poor prognosis in HL correlate with tumor invasion and metastasis. Hodgkin and Reed-Sternberg (H/RS) cells, although only accounting for less than $1 \%$ of total HL tumor cells, contribute considerably to tumor progression [1]. The mechanism may involve immune escaping which was conducted by interaction of various cytokines and chemokines between tumor cells and inflammatory cells in the tumor microenvironment [2].

Accumulating evidence suggests that chemokine ligands interact with corresponding receptors to induce a variety of cell responses that affect tumor progression, such as cell proliferation, migration, invasion and metastasis. CXCL16 is a chemokine that exists in two forms: a transmembrane form (TM-CXCL16) and a soluble form (sCXCL16) which is secreted into the cell culture supernatant via the cleavage of TM-CXCL16 by a disintegrin-like metalloproteinase 10 (ADAM10) [3]. Both forms of CXCL16 interact with its unique receptor CXCR6 to exert distinct functions [4,5]. Interaction of SCXCL16 with CXCR6 has been reported in various tumors and is associated with tumor growth, metastasis, and reoccurrence [5-7]. Darash-Yahana [8] and Deutsch [9] highlighted that CXCL16 and CXCR6 may be expressed in 
lymphoma, however, the role of the CXCL16-CXCR6 axis in lymphatic malignancies is yet to be elucidated. In our previous study, CXCL16 expression was found negatively correlated with the expression of CD99 in human and mouse lymphoma cell lines [10,11]; besides, CXCL16 were found highly expressed in several lymphoma cell lines as well as in clinical samples [11]. In diffuse large B cell lymphoma (DLBCL), co-expression of CXCL16 and CXCR6 is a risk factor for poor prognosis of patients with diffuse large B Cell lymphoma [12], while in HL, the function of CXCL16 on H/RS cells and the involved pathways needs further clarification. The present study aims to characterize the effect of sCXCL16 on the phenotypes of H/RS cells in vitro and to explore the role of the PI3K/Akt/NF- $\kappa B$ pathways in the H/RS cell progression regulated by sCXCL16-CXCR6 axis, which may provide experimental data for potential strategy in clinical therapy.

\section{Methods}

\section{Cell culture and transfection}

L428 cells were cultured in RPMI-1640 medium (Gibco BRI, Gaithersburg, MD) supplemented with 10\% heat-inactivated fetal bovine serum (FBS; Hyclone Technologies, Logan, UT) at 37 ${ }^{\circ} \mathrm{C}$ in a humidified atmosphere with $5 \% \mathrm{CO}_{2}$. A CXCL16 lentivirus vector, CXCL16-pGC FU-GFP-LV, was established by recombinating the CXCL16 gene with empty lentivirus vector (pGC FU-GFP$\mathrm{LV}$ ), identified, then transfected into L428 cells. The subclone overexpressed CXCL16 was named as CXCL16+ group. Normal L428 cells and L428 cells transfected with empty lentivirus vector were used as controls (hereafter referred to as Control-1 and Control-2, respectively). Cell morphology and Green Fluorescence Protein (GFP) were routinely observed using fluorescence microscopy. Stable expression and secretion of sCXCL16 in different cell groups were validated using quantitative RT-PCR, western blot analysis, and Enzyme-Linked Immunosorbent Assays (ELISA).

\section{RNA isolation and quantitative RT-PCR}

RNA isolation reagent was mixed with different groups of cells at a concentration of $5 \times 106$ cells $/ m L$. Total RNA isolation and cDNA generation were performed as previously described [10]. The sequences of primers used were as follows: CXCL16: forward: 5'-CGTCACTGGAAGTTGTTATTGTGGT-3'; reverse: 5'-TGGTAGGAAGTAAATGCTTCTGGTG-3'; GAPDH: forward: 5'-GCTGAGTATGTCGTGGAGTCT-3'; reverse: 5'-GTTCACACCCATCACAAACAT-3'. GAPDH was used as an internal control. The relative expression ratio of CXCL16 was calculated using the 2- $\Delta \Delta \mathrm{Ct}$ method.

\section{Enzyme-linked immunosorbent assay}

Cells were cultured in FBS-free RPMI 1640 medium at a concentration of $1 \times 106$ cells/mL for $8 \mathrm{~h}$ followed by centrifugation at $1500 \mathrm{rpm}$ for $5 \mathrm{~min}$. Commercially available ELISA kits (Becton Dickinson, Franklin Lakes, NJ) were used according to the manufacturer's instructions to measure CXCL16 concentrations at $450 \mathrm{~nm}$.

\section{Cell proliferation assay}

Cell proliferation in two control groups, CXCL16+ group (L428 cells transfected with CXCL16-pGC FU-GFP-LV), sCXCL16 group (L428 cells treated with $100 \mathrm{ng} / \mathrm{mL}$ recombinant sCXCL16), and anti-CXCR6 group (L428 cells treated with 100ng/mL CXCR6 antibody) was examined using Cell Counting Kit-8 (CCK-8; Tongren Shanghai Co., China), as previously described [11]. ThesCXCL16 recombinant protein and CXCR6 antibody were purchased from Peprotech, Rocky Hill, NJ. The proliferation of L428 cells upon treatment with the PI3K/Akt inhibitor LY294002 at concentrations ranging from 0 to $50 \mu \mathrm{M} / \mathrm{mL}$ was also measured.

\section{Transwell assays}

Transwell migration assays were performed in 24-well insert plates (Corning Costar, Tewksbury, MA). First, $200 \mu \mathrm{L}$ of cells in serum-free medium were added to the upper chambers $(5 \mu \mathrm{m}$ pore size) and placed into wells containing $500 \mu \mathrm{L}$ medium supplemented with $10 \%$ FBS at $1 \times 105$ cells/well. The plate was then incubated for $4 \mathrm{~h}$ at $37^{\circ} \mathrm{C}$. Cell migration to the bottom chamber was examined using CCK-8 as described above.

\section{Flow cytometry}

For cell cycle and apoptosis analysis, the cell samples were cultured and treated using commercially available cell cycle (Kaiji Co., China) or apoptosis (Dojindo Molecular Technologies, Inc., Japan) kits according to the manufacturer's instructions and subjected to flow cytometry analysis using a FACS Vantage flow cytometer with CellQuest acquisition and analysis software (Becton Dickinson, Franklin Lakes, NJ). The cell samples were collected after $24 \mathrm{~h}$ of culturing and resuspended in $1 \times$ PBS containing antibodies against CD15, CD30, CD19 or CD20, incubated for 30 min in the dark, and subjected to flow cytometry analysis.

\section{Protein extraction and western blot analysis}

Protein extraction and western blot analysis were performed as previously described [10]. Briefly, L428 cells were collected at different time points $(0,15,30,45$, and $60 \mathrm{~min})$ after treatment with $100 \mathrm{ng} / \mathrm{mL}$ of recombinant sCXCL16. Total proteins were examined on $10 \%$ SDS-PAGE gel and electro transferred onto Polyvinylidene Fluoride (PVDF) membranes (Merck Millipores, Burlington, MA). The membranes were blocked with 5\% skim milk and incubated with primary and secondary antibodies (Cell Signaling Technology, Danvers, MA) with an enhanced chemiluminescence system to reveal specific bonds. Images were captured and analyzed using a Gel Documentation System (Bio-Rad, Hercules, CA).

\section{Statistical analysis}

All images obtained from western blotting and flow cytometry experiments were representative of at least three independent experiments. The data shown were expressed as means \pm SD. Statistical analysis were performed using SPSS 17.0 software. Data were compared using one-way ANOVA, and p-values $<0.05$ were considered statistically significant. 


\section{Results}

\section{sCXCL16 promoted proliferation and migration of H/RS cells}

The CXCL16 gene was cloned into the lentivirus vector pGC FU-GFP-LV, then transfected L428 cells. Stable expression was achieved and the secretion of sCXCL16 was assessed (Figure 1). The proliferation of different cell groups was investigated by CCK8 assays. Results showed that sCXCL16 significantly promoted the proliferation of H/RS cell line (Figure 1). Transwell assays demonstrated that sCXCL16 promoted the migration of L428 cells in both CXCL16+ group, which endogenously overexpressed CXCL16, and sCXCL16 group, in which $100 \mathrm{ng} / \mathrm{mL}$ recombinant sCXCL16 was applied to L428 cells (Figure 1). To test whether binding to its receptor CXCR6 was essential for the effect of endogenous CXCL16 on cell proliferation and migration, CXCR6 antibody was applied at a concentration of $100 \mathrm{ng} / \mathrm{mL}$. Blocking CXCR6 effectively slowed down cell proliferation and migration (Figure 1).

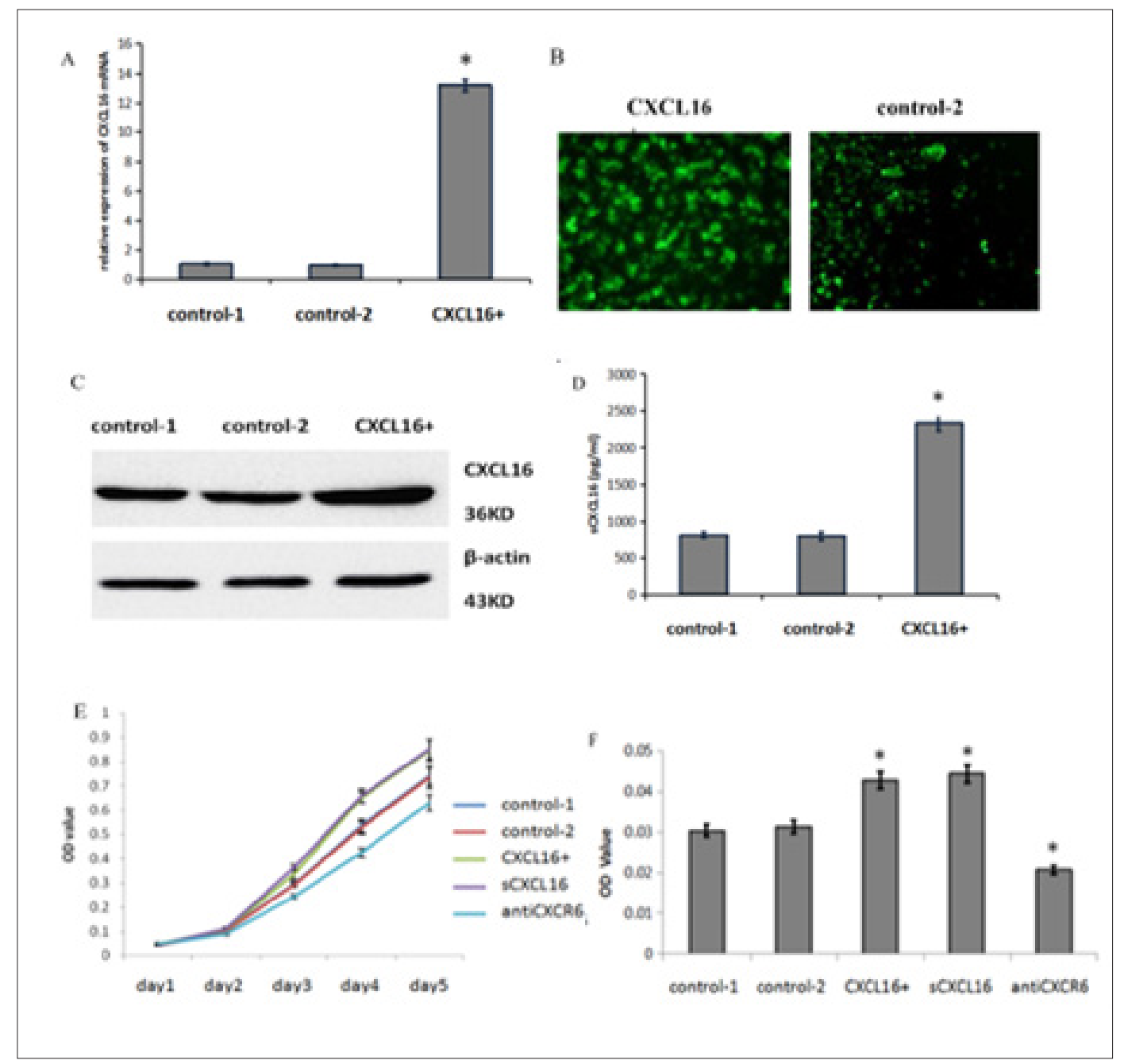

Figure 1: CXCL16 promote cell proliferation and migration of Hodgkin Reed-Sternberg cells.

A. The expression levels of CXCL16 mRNA were analysed by quantitative RT-PCR in Hodgkin Reed-Sternberg cell line L428 (control-1 group), L428 cell line transfected with empty lentivirus vector (control-2 group) and CXCL16-pGC FU-GFP-LV lentivirus vector (CXCL16+ group).

B. GFP was observed by fluorescence microscopy $120 \mathrm{~h}$ after transfection.

C. The expression of CXCL16 and $\beta$-actin in different groups were analysed by western blotting.

D. Soluble CXCL16 in the supernatant of cultured L428 cells were observed detected using ELISA. Proliferation

E. Chemotaxis

F. L428 cells under different treatments were assessed. 100ng/mL recombinant sCXCL16 or anti-CXCR6 were added to control-1 cells respectively. Data were expressed as the mean \pm SD and compared by one-way ANOVA. Asterisk (*) denotes significant differences $(\mathrm{p}<0.05)$ between control and treated cells. 


\section{sCXCL16 affected cell cycle of H/RS cells and suppressed apoptosis}

Flow cytometry assays were performed to investigate the effect of sCXCL16 on cell cycle and apoptosis in different cell groups. The percentage of cells in G1 phase was considerably lower in the CXCL16+ group ( $42.40 \pm 1.18 \%$ ) and sCXCL16 group (42.26 $\pm 1.36 \%)$ as compared with the control groups (56.58 $\pm 0.83 \%)$, while the percentage of cells in G2/M phase was higher in the CXCL16+ group $(27.32 \pm 0.2 \%)$ and sCXCL16 group $(27.6 \pm 2.25 \%)$ than that of the control groups $(16.39 \pm 1.37 \% ; \mathrm{p}<0.05)$. In contrast, treatment of normal L428 cells with CXCR6 antibody significantly increased the percentage of G1 phase cells and reduced the percentage of G2/M phase cells $(\mathrm{p}<0.05)$ (Figure 2).

Apoptosis assays demonstrated that the percentage of apoptotic cells was diminished in the CXCL16+ and sCXCL16 groups compared with the control groups and was dramatically elevated by over 2-fold when CXCR6 antibody was applied to the normal L428 cells, the CXCL16+ group and the SCXCL16 group (Figure 2).

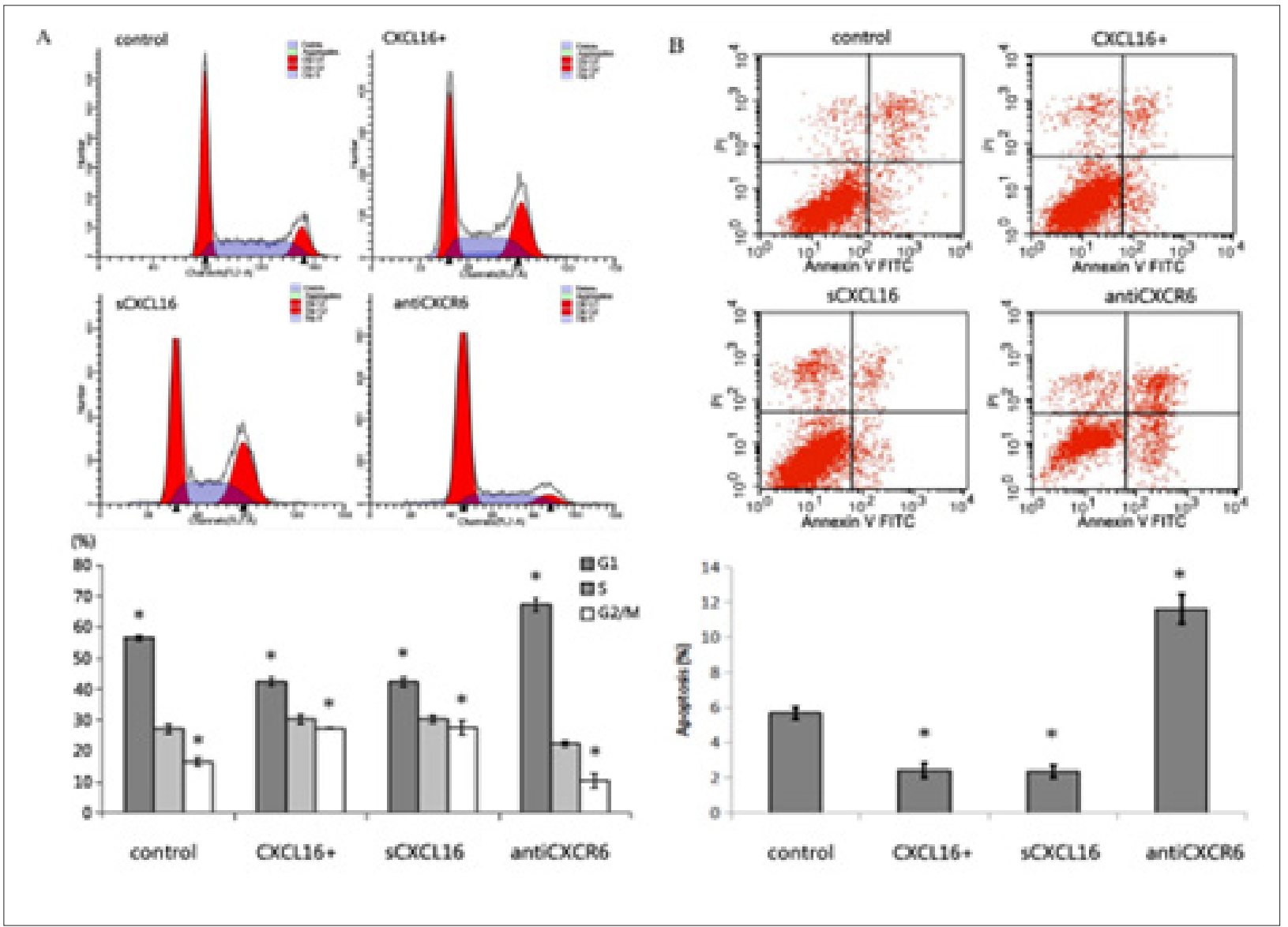

Figure 2: sCXCL16-mediated cell cycle change and apoptosis of Hodgkin Reed-Sternberg cells.

A. Cell cycles were measured to test the effects of overexpression of CXCL16 and the addition of $100 \mathrm{ng} / \mathrm{mL}$ recombinant sCXCL16 or anti-CXCR6 on L428 cells. Histograms represent the percentage of L428 cells detected at different cell cycle stages.

B. Apoptosis was measured at $24 \mathrm{~h}$ intervals after treatments in each group. Histograms represent the percentage of apoptotic L428 cells. Flow cytometry results represent at least three independent experiments. Data were expressed as the mean \pm SD and compared by one-way ANOVA. Asterisk (*) indicates significant differences $(p<0.05)$ between control and treated cells.

\section{sCXCL16 did not change the immune phenotypes of $\mathrm{H} /$ RS cells}

Flow cytometry was performed to detect immune phenotypes in different cell groups. Expressions of CD19 and CD20, both are biomarkers of B-cell lymphoma instead of HL [13], were rather low, while CD15 and CD30, which are biomarkers of HL, were highly expressed in all groups (Figure 3). There were no substantial differences in immune phenotypes observed among different groups upon CXCL16 treatment ( $p>0.05)$, indicating that neither overexpression of CXCL16 or exogenously applied sCXCL16 affect the immune phenotypes of $\mathrm{H} / \mathrm{RS}$ cells in vitro. 


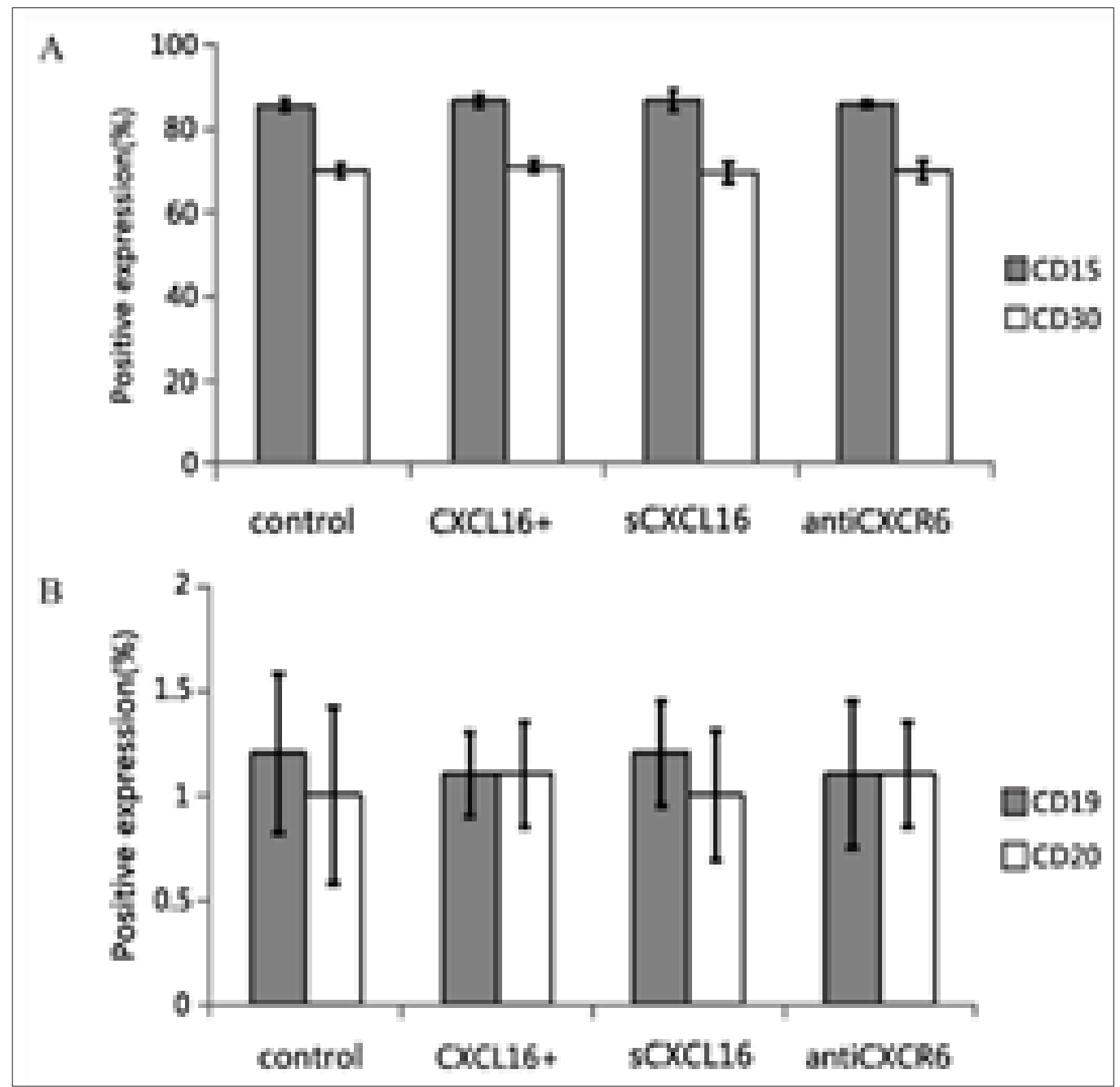

Figure 3: The immune phenotypes of L428 by applying recombinant sCXCL16.

Expression of immune phenotype markers (A) CD15, CD30, (B) CD19 and CD20 in L428 cells were measured using flow cytometry at $24 \mathrm{~h}$ after treatments. Data were expressed as the mean $\pm \mathrm{SD}$ and compared by one-way ANOVA. * $\mathrm{p}<0.05$ vs control.

sCXCL16 regulated the PI3K/Akt/NF-אB pathway in $\mathrm{H} /$ RS cells

The PI3K/Akt/NF- $\mathrm{B}$ signaling pathway plays a crucial role in regulating physiological and pathological events in cells. Dysfunction of these pathways contribute to tumor progression. Our previous studies have shown that CXCL16, including TMCXCL16 and sCXCL16, are highly expressed in several lymphoma tissues and cell lines [11]. Elevated sCXCL16 expression was correlated with activation of the NF- $\mathrm{BB}$ pathway in the H/RS cell line L428 [11]. However, the mechanism of the effects of CXCL16 on H/RS cells is not clear. To determine whether sCXCL16 could promote the activation of the PI3K/Akt/NF- $\mathrm{KB}$ signaling pathways, western blot analysis was performed. Data showed that in the presence of SCXCL16, the expressions of phospho-Akt, phosphop70S6, and phospho-IкB were increased in a time-dependent manner, with maximum expression level reached at 45 and 60 min after treatment with 100ng/mL recombinant sCXCL16 (Figure 4). In addition, both CXCL16 overexpression and exogenously applied recombinant sCXCL16 increased the phosphorylation of Akt, p70s6, and IкB proteins, while blockade of CXCL16 or CXCR6 by antibodies decreased the phosphorylation of these proteins in L428 cells, suggesting that the CXCL16-CXCR6 interaction may lead to the activation of the PI3K/Akt/NF- $\mathrm{KB}$ pathways (Figure 4).

To further validate the results, LY294002, an inhibitor of PI3K signaling pathway, was applied. The results showed that LY294002 inhibited L428 cell proliferation and migration in a dose-dependent manner, with the strongest effect observed at concentration of $50 \mu \mathrm{M} / \mathrm{mL}$ (Figure 4). In addition, the presence of LY294002 effectively suppressed sCXCL16-induced cell proliferation and migration (Figure 4). These data provide further evidence that the functions of SCXCL16 in L428 cells are at least partially mediated through the PI3K/Akt/NF- $\mathrm{BB}$ signaling pathway. 

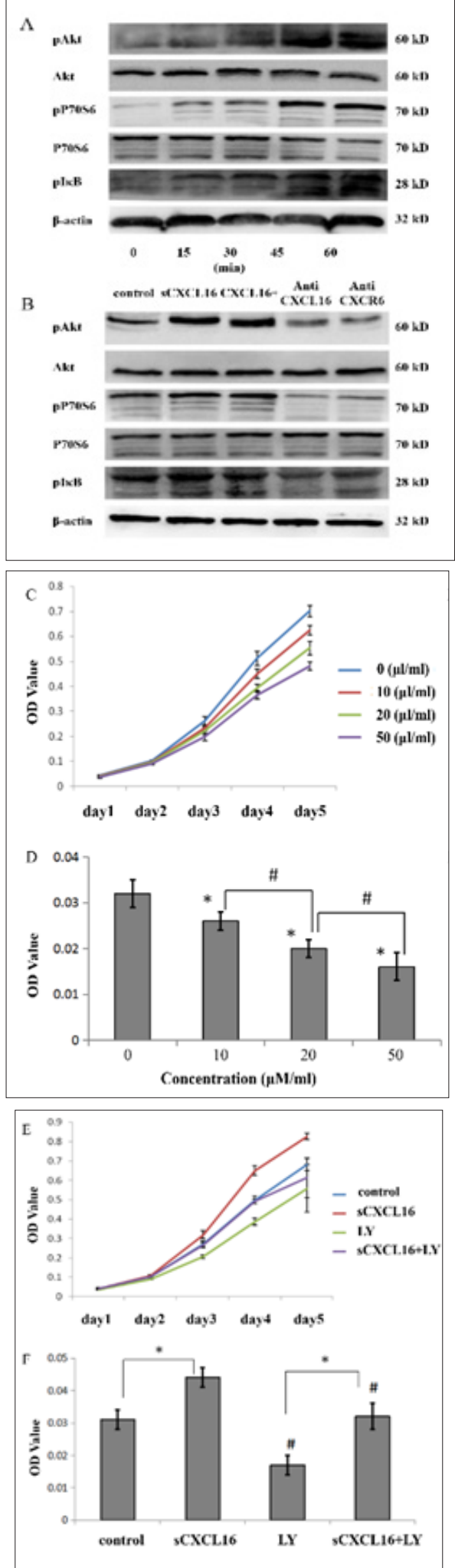

Figure 4: sCXCL16-mediated cell proliferation and migration in L428 cells through activation of the PI3K/ Akt/NF-kB pathway. A-B: Western Blot analysis of expression of PI3K/Akt/NF-kB pathway-related proteins. Time-dependent effects of $100 \mathrm{ng} / \mathrm{mL}$ recombinant sCXCL16 on several groups were observed in CXCL16 overexpressed cell line (A) and 100ng/mL recombinant sCXCL16 exogenously applied group (B). Effect of PI3K inhibitor LY294002 (LY) on the proliferation and migration of different groups were investigated: The concentrationdependent effects of LY294002 on the L428 cells (C, D) and effect of $100 \mathrm{ng} / \mathrm{mL}$ recombinant sCXCL16 on the L428 cells (E-F). Data were expressed as the mean \pm SD and compared by one-way ANOVA. *p $<0.05$ vs control. 


\section{Discussion}

HL is an inflammation-associated tumor. H/RS cells, the presence of which is a hallmark of HL, secrete various cytokines and chemokines into the tumor microenvironment, contributing to development and progression of HL. Chemokines such as CXCL9 [14], CXCL10 [15], CCL5[16], CCL17, and CCL22 [17] have been reported to participate in the crosstalk between H/RS and inflammatory cells by binding to corresponding receptors, and is associated with tumor cell growth, proliferation, migration, immunosuppression, and poor prognosis.

CXCL16 was first described in inflammatory and immune diseases [18] and was found in recent years to be correlated with tumor progression in several cancer types, including prostate cancer [5], lung cancer [7], ovarian cancer [6], and gastric cancer. Elevated CXCL16 expression has been reported to have the potential to be an independent predictor of poor clinical outcomes in cancer $[19,20]$. CXCL16 binds to its unique receptor, CXCR6, to induce downstream physiological and pathological outcomes. In the last decade, more evidence shows that CXCL16-CXCR6 axis could not only be a potential biomarker but an important target of clinical therapy [21-23].

Our group previously reported that CXCL16 was highly expressed in clinical tissues of HL patients and in nine human lymphoma cell lines [11] and was identified as a risk factor for poor prognosis in diffuse large B-cell lymphoma [12]. As high expression of CXCR6 was also detected in the HL cell line (L428) [11], we wondered whether regulation of CXCL16 would have any effect on H/RS cell phenotype and its extensive signaling pathways. In the present study, we used two methods to investigate the effects of CXCL16 on L428 cell lines. One was to construct a CXCL16 overexpressed lentivirus vector and transfect it into L428 cells. The second was to apply recombinant SCXCL16 protein to the cultured L428 cells. Results showed that both endogenous overexpressed CXCL16 and exogenously applied recombinant sCXCL16 promoted L428 cell proliferation and migration, and significantly suppressed cell apoptosis; meanwhile, CXCR6 antibody effectively reversed these effects, suggesting that sCXCL16 influences target cells through interaction with its unique receptor, CXCR6. Therefore, it is proposed that sCXCL16 may have an autocrine effect by coexpression with CXCR6 on H/RS cells. These results are consistent with previous studies that the CXCL16-CXCR6 axis could promote cell proliferation and migration in various tumor cells $[7,20]$.

As the signal pathways were concerned, it is well known that the PI3K/Akt/NF- $\mathrm{KB}$ signaling pathway is strongly correlated with tumor progression and considered to be an effective target in cancer chemotherapy. The CXCL16-CXCR6 axis has been reported to promote tumor cell proliferation, invasion, and metastasis via up-regulation of the PI3K/Akt pathway in pancreatic cancer [24] and ovarian cancer [6], and via the NF- $\kappa B$ pathway in prostate cancer [5]. In gastric cancer, blockade of CXCR6 suppressed tumor growth through inhibition of Akt signaling [25]. In HL, reports showed that constitutive activation of PI3K/Akt/mTOR pathway contributed to cell survival [26]; downregulation of PI3K by
LY209002 [27] or RP6530 (a dual PI3K / $\gamma$ inhibitor) [28] promoted G1 cell cycle arrest and cell apoptosis, leading to attenuated HL cell proliferation. Our previous investigation revealed that sCXCL16 was correlated with NF- $\kappa B$ activation in HL [11]. The present results showed that SCXCL16 activated the PI3K/Akt/NF- $\mathrm{B}$ pathways in a time-dependent manner. Blockade of CXCL16 or CXCR6 in L428 cells reduced the activation of $\mathrm{PI} 3 \mathrm{~K} / \mathrm{Akt} / \mathrm{NF}-\kappa \mathrm{B}$ pathway, and application of LY294002 significantly diminished sCXCL16-induced H/RS cell proliferation and migration. Together, these results provide evidence that activation of the PI3K/Akt/NF- $\mathrm{KB}$ signaling pathway was possibly involved in the autocrine effect of sCXCL16 in promoting H/RS cell progression.

Immune phenotypes are important markers in different lymphoma sub-types. CD15 and CD30 are expressed in almost all $\mathrm{H} / \mathrm{RS}$ cells and are commonly used as diagnostic markers for HL [29]; meanwhile, CD19 and CD20 are used to diagnose lymphoma derived from B-cell [13]. Our previous study demonstrated that up-regulation of the cell differentiation antigen CD99, which is inversely correlated with CXCL16 expression, significantly influenced immune phenotype in L428 cells; specifically, it led to the loss of cell surface markers CD15 and CD30 [11], and stimulated phenotype transformation from H/RS into B-cells. While in the present study, results revealed that sCXCL16 did not have an impact on immune phenotypes of $\mathrm{L} 428$ cells, indicating that differentiation or transformation of H/RS cells were not affected by CXCL16.

In summary, it is discovered in the current study that sCXCL16 promoted proliferation and migration of H/RS cells in vitro, which could be partially mediated by the activation of the PI3k/Akt/NF$\kappa B$ signaling pathways. These findings suggest that inhibition of sCXCL16 or CXCR6 may serve as a potential strategy for therapeutic HL.

\section{Consent for Publication}

This paper has not been published elsewhere in whole or in part. All authors have read and approved the content and agree to submit it for consideration for publication.

\section{Competing interests}

All authors declare no competing interests.

\section{Funding}

This work was supported by funding of the National Key Research and Development Program (2018YFA0902702) and the National Science and Technology Major Project (2018ZX10731301003-007); National Natural Science Foundation of China (81801558, 81870307), Basic and applied basic research project of Guangdong Province (2019A1515110495), Guangdong Medical Research Fund (A2019396), and Science and Technology Project of Shenzhen City (JCYJ20160427190318822).

\section{Authors' Contributions}

Conception and design: F.Liu; development of methodology: Y.Xun, H.Tang; experiments: H.Tang, Y.Xun, Y. Liang, H. Yang, D. Shen; analysis and interpretation of data: L. Fan, R. Wang, H. Liu; writing, 
review, and/or revision of the manuscript: Y. Xun, F. Liu, Y. Pan; technical/material support: Y. Ai. D. Liu; study supervision: F. Liu, H. Tang. All authors read and approved the final manuscript.

\section{Acknowledgement}

The authors thank Professor Tong Zhao from Guangdong Provincial Key Laboratory of Molecular Oncopathology, Southern Medical University for kindly providing H/RS cell line L428.

\section{References}

1. Piris MA, Medeiros LJ, Chang KC (2020) Hodgkin lymphoma: A review of pathological features and recent advances in pathogenesis. Pathology 52(1): 154-165.

2. Xun Y, Yang H, Li J, Wu F, Liu F (2020) CXC chemokine receptors in the tumor microenvironment and an update of antagonist development. Rev Physiol Biochem Pharmacol 178: 1-40.

3. Yang H, Qiu B, Chen S, Xun Y, Pan Y, et al. (2019) Soluble CXCL16 promotes TNF-alpha-induced apoptosis in DLBCL via the AMAD10-NF-kappaB regulatory feedback loop. Cell Biol Int 43(8): 863-874.

4. Chung B, Esmaeili A, Gopalakrishna S, Murad P, Andersen E, et al. (2017) Human brain metastatic stroma attracts breast cancer cells via chemokines CXCL16 and CXCL12. NPJ Breast Cancer 3: 6.

5. Kapur N, Mir H, Guru P, Sanjay J, Bae S, et al. (2019) Prostate cancer cells hyper-activate CXCR6 signaling by cleaving CXCL16 to overcome effect of docetaxel. Cancer Lett 454: 1-13.

6. Hong L, Wang S, Li W, Wu D, Chen W, et al. (2018) Tumor-associated macrophages promote the metastasis of ovarian carcinoma cells by enhancing CXCL16/CXCR6 expression. Pathol Res Pract 214(9): 13451351.

7. Liang K, Liu Y, Ere D, Liu J, Yang F, et al. (2018) High CXC Chemokine Ligand 16 (CXCL16) expression promotes proliferation and metastasis of lung cancer via regulating the NF-kappa B pathway. Med Sci Monit 24: 405-411.

8. Darash-Yahana M, Gillespie W, Stephen M, Yun K, Shin M, et al. (2009) The chemokine CXCL16 and its receptor, CXCR6, as markers and promoters of inflammation-associated cancers. PLoS One 4(8): e6695.

9. Deutsch AJ, Steinbauer E, Hofmann N, Strunk D, Gerlza T, et al. (2013) Chemokine receptors in gastric MALT lymphoma: Loss of CXCR4 and upregulation of CXCR7 is associated with progression to diffuse large B-cell lymphoma. Mod Pathol 26(2): 182-94.

10. Liu F, Zhang G, Liu F, Zhou X, Xiaoyan C, et al. (2013) Effect of shRNA targeting mouse CD99L2 gene in a murine B cell lymphoma in vitro and in vivo. Oncol Rep 29(4): 1405-1414.

11. Liu F, Zhang Y, Hongping T, Xinhua Z, Ziqing W, et al. (2013) CXC chemokine ligand 16, inversely correlated with CD99 expression in Hodgkin Reed-Sternberg cells, is widely expressed in diverse types of lymphomas. Oncol Rep 30(2): 783-792.

12. Qiu B, Liu F, Tang H, Liu J, Liu L, et al. (2016) Co-expression of CXCL16 and CXCR6 is a risk factor for poor prognosis of patients with diffuse large B Cell lymphoma. Int J Clin Exp Pathol 9(5): 5368-5377.

13. Martyniszyn A, Ann-Christin Krahl, Maya André, Hombach A, Abkeri H, et al. (2017) CD20-CD19 bispecific CAR T cells for the treatment of B-cell malignancies. Hum Gene Ther 28(12): 1147-1157.

14. Vassilakopoulos TP, Georgia L, Vassilis M, Hartmann S, Lakiotaki E, et al. (2017) Thioredoxin-1, chemokine (C-X-C motif) ligand-9 and interferongamma expression in the neoplastic cells and macrophages of Hodgkin lymphoma: clinicopathologic correlations and potential prognostic implications. Leuk Lymphoma 58(9): 1-13.

15. Chiu J, Ernst DM, Keating A (2018) Acquired Natural killer cell dysfunction in the tumor microenvironment of classic hodgkin lymphoma. Front Immunol 9: 267.

16. Casagrande N, Cinzia B, Lydia V, Maurizio M, Colombatti A, et al. (2019) CCR $_{5}$ antagonism by maraviroc inhibits Hodgkin lymphoma microenvironment interactions and xenograft growth. Haematologica 104(3): 564-575.

17. Goncharova O, Flinner N, Bein J, Döring C, Donnadieu E, et al. (2019) Migration properties distinguish tumor cells of classical hodgkin lymphoma from anaplastic large cell lymphoma cells. Cancers (Basel)11(10): 1484.

18. Morgan AJ, Guillen C, Symon FA, Huynh TT, Berry MA, et al. (2005) Expression of CXCR6 and its ligand CXCL16 in the lung in health and disease. Clin Exp Allergy 35(12): 1572-1580.

19. Richardsen E, Nora N, Melbø-Jørgensen C, Johannesen C, Grindstad T, et al. (2015) The prognostic significance of CXCL16 and its receptor C-X-C chemokine receptor 6 in prostate cancer. Am J Pathol 185(10): 27222730 .

20. Lang K, Bonberg N, Robens S, Behrens T, Hovanec J, et al. (2017) Soluble chemokine (C-X-C motif) ligand 16 (CXCL16) in urine as a novel biomarker candidate to identify high grade and muscle invasive urothelial carcinomas. Oncotarget 8(62): 104946-104959.

21. Ikeda T, Nishita M, Hoshi K, Honda T, Yoshihiro K, et al. (2020) Mesenchymal stem cell-derived CXCL16 promotes progression of gastric cancer cells by STAT3-mediated expression of Ror1. Cancer Sci 111(4): 1254-1265.

22. Mir H, Neeraj K, Gales D, Praveen K, Gabriela O, et al. (2021) CXCR6CXCL16 axis promotes breast cancer by inducing oncogenic signaling. Cancers (Basel)13(14): 3568.

23. Karaki S, Charlotte B, Thi Tran, Isabelle G, Alice M, et al. (2021) CXCR6 deficiency impairs cancer vaccine efficacy and CD8(+) resident memory T-cell recruitment in head and neck and lung tumors. J Immunother Cancer 9(3): e001948.

24. Chalabi-Dchar M, Stéphanie C, Camille D, Marjorie F, Hubert L, et al. (2015) Loss of somatostatin receptor subtype 2 promotes growth of KRAS-induced pancreatic tumors in mice by activating PI3K signaling and overexpression of CXCL16. Gastroenterology 148(7): 1452-1465.

25. Li Y, Li-Xia Fu, Wan-Lin Zhu, Hua Shi, Li-Jian Chen, et al. (2015) Blockade of CXCR6 reduces invasive potential of gastric cancer cells through inhibition of AKT signaling. Int J Immunopathol Pharmacol 28(2): 194200.

26. Dutton A, Reynolds G, Dawson C, Young S, Murray P, et al. (2005) Constitutive activation of phosphatidyl-inositide 3 kinase contributes to the survival of Hodgkin's lymphoma cells through a mechanism involving Akt kinase and mTOR. J Pathol 205(4): 498-506.

27. Georgakis GV, Yang Li, Rassidakis G, Medeiros J, Mills G, et al. (2006) Inhibition of the phosphatidylinositol-3 kinase/Akt promotes G1 cell cycle arrest and apoptosis in Hodgkin lymphoma. Br J Haematol 132(4): 503-511.

28. Locatelli SL, Giuseppa C, Simone S, Consonni F, Maeda A, et al. (2019) Targeting cancer cells and tumor microenvironment in preclinical and clinical models of hodgkin lymphoma using the dual PI3Kdelta/gamma inhibitor RP6530. Clin Cancer Res 25(3): 1098-1112.

29. O’Malley DP, Dogan A, Fedoriw Y, Medeiros J, Chi Young, et al. (2019) American registry of pathology expert opinions: Immunohistochemical evaluation of classic Hodgkin lymphoma. Ann Diagn Pathol 39: 105-110. 